\title{
Ovarian function, fertility and reproductive lifespan in cancer patients
}

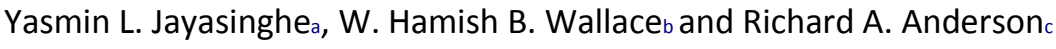

aDepartment of Obstetrics and Gynaecology, Royal Women's Hospital, University of Melbourne, Melbourne, Australia; bDepartment of

Haematology and Oncology, Royal Hospital for Sick Children, Edinburgh, UK; cMRC Centre for Reproductive Health, Queens Medical Research

Institute, University of Edinburgh, Edinburgh, UK

\begin{abstract}
Introduction: The increasing survival of girls and young women after cancer has led to a rapid growth in research into assessment of ovarian function after treatment.
\end{abstract}

Areas covered: This aim of this review is to discuss normal ovarian function over time, the impact of cancer treatment on ovarian function, the assessment of ovarian reserve after treatment, and pretreatment predictors of ovarian recovery.

Expert commentary: Ovarian function damage after chemotherapy and radiotherapy will impact on fertility and reproductive lifespan, but with great variability. Age at menopause has implications for the duration of estrogen deficiency, with its own adverse health consequences. This has led to identification of the key treatment and patient factors at the time of treatment, notably age and ovarian reserve that impact on post-treatment ovarian function. However, most studies have used outcome measures such as ongoing menses, or biomarkers such as anti-mullerian hormone $(\mathrm{AMH})$, with few reporting on fertility or age at menopause.

\section{Key words}

antimullerian hormone/chemotherapy / ovarian reserve / radiotherapy / premature ovarian insufficiency 


\subsection{Introduction}

Worldwide, around 1.7 million girls and women under 50 years are diagnosed with cancer annually [1], with $5 \%$ affecting young people $\leq 35$ years, in their reproductive prime. Due to advances in treatment, improvements in life expectancy have resulted in a growing population of survivors but these treatments can adversely affect ovarian function across the lifespan, causing pubertal failure, infertility, premature ovarian insufficiency (POI), and health sequelae due to chronic oestrogen deficiency [2]. Compromised fertility is the most prevalent long-term side effect of cancer therapy [3] and affects long-term wellbeing, relationships and life decisions [4]. Ovarian function and reproductive capacity after cancer treatment are important quality of life issues, but many survivors are poorly informed about their fertility status $[5,6]$ and regular menses may be falsely reassuring [7]. Ovarian reserve testing, where several candidate markers are examined to indirectly estimate a woman's reproductive potential, is being rapidly adopted, however, there is no gold standard 'fertility test' [8]. This review will discuss normal ovarian function over time, the impact of cancer treatment on ovarian function, the assessment of ovarian reserve after treatment, and pretreatment predictors of ovarian recovery.

\subsection{Ovarian function with age}

The primordial follicle pool constitutes the ovarian reserve, which is a measure of the ability of the ovary to produce mature eggs which can be fertilised [9]. Follicular renewal has not been established to date, and it is accepted that the ovarian reserve is finite and complete before birth. Modelling studies based on histological analysis demonstrate a peak in the primordial follicle pool at $18-22$ weeks, with an average of 295000 primordial follicles per 
ovary at birth (95\% prediction interval 33,800-2,508,000), declining to $180000[21,300-$ $1,512,000]$ at 13 years [10] (figure 1).

Recruitment of follicles occurs across the reproductive lifespan [11]. In the prepubertal girl follicle activation and maturation reaches primary, pre-antral and even early antral phases, with development to later antral stages and ovulation from puberty following increases in secretion of follicle stimulating hormone (FSH) and luteinising hormone (LH) from the pituitary. Growth activation of primordial follicles peak at around 14 years then subsequently declines. By 30 years of age, around $12 \%$ of the ovarian reserve remains, which declines to $3 \%$ by age 40 years [10]. Only around 450 follicles will ovulate in the reproductive lifetime, thus the majority of follicles, at any stage of development, undergo atresia, until insufficient follicles remain that can develop to later stages, resulting in the menopause at around 50 years $[12,13]$. Age contributes to around $80 \%$ of the variation in primordial follicle numbers, and is also the key determinant of reduced oocyte quality. Other determinants include genetic and lifestyle factors (stress, parity, basal metabolic index and smoking), the latter being thought to make a 3-5\% contribution to age of menopause $[14,15]$.

Regulation of early follicle growth is through a balance between activating and inhibitory autocrine and paracrine factors. A major regulatory pathway within the oocyte is the phosphatidylinositol-3'-kinase (PI3K-AKT) signalling pathway. The phosphatase and tensin homolog deleted on chromosome 10 (PTEN) and transcription factor FOXO3 suppress follicle activation [16]. Local paracrine inhibitory factors include anti-Mullerian hormone (AMH), which is a principal regulator of folliculogenesis by inhibiting the primordial to primary follicle transition [17]. AMH is produced by the granulosa cells of preantral and 
small antral follicles, (the latter being the main source of $\mathrm{AMH}$ ) [18], with expression falling sharply when follicles reach $8-10 \mathrm{~mm}$ diameter. $\mathrm{AMH}$ levels correlate with the antral follicle count [19], and while not produced by non-growing follicles, are an indirect measure of the size of the primordial follicle pool.

AMH levels across the lifespan reflect non-growing follicle recruitment $[20,21,22]$. AMH is present at low levels in prepubertal females, and increases to a peak at around 25 years [20, 22]. AMH levels demonstrate two small dips, the first soon after birth (following the brief rise of neonatal minipuberty), and again at the start of puberty for a couple of years before rising again $[20,21,22]$. In women $\geq 25$ years $\mathrm{AMH}$ follows a non-linear decline, inversely correlated with age [20]. AMH becomes undetectable before the menopause: initial studies indicated that this occurred approximately 5 years before the menopause [23]; current automated assays have much improved sensitivity thus this is gap is likely to be shortened [24].

The prepubertal ovary also contains a population of apparently abnormal primordial follicles, characterised by larger size and indistinct germinal vesicles, which are not present in the adult human ovary [25]. Follicles isolated from prepubertal and adolescent girls also have intrinsically reduced capacity for growth. Thus it appears that there is more to postnatal ovarian development than previously recognised, but the clinical significance of these findings is unclear. Understanding of the regulation of early follicle growth is more limited than that of later gonadotropin dependant stages, but is important for analysis of the effects of gonadotoxic therapy on the ovary, and the changes in follicle growth dynamics that result. Advances in this field might lead to therapeutic developments, illustrated by the potential for the use of $\mathrm{AMH}$ ligands as potential chemoprotectants [26]. 


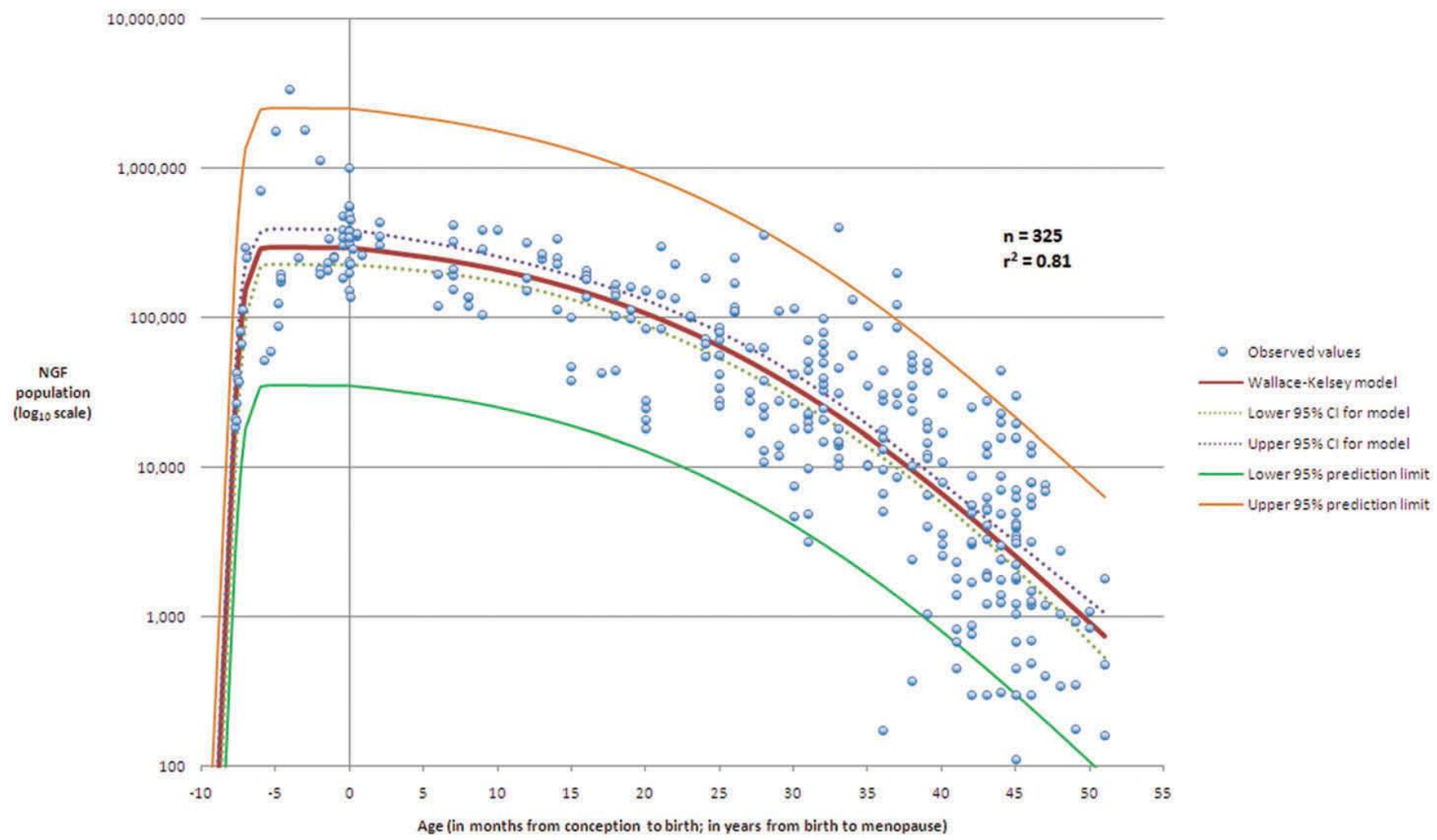

Figure 1. Normative model for the non-growing follicle (NGF) population. The best model for the establishment of the NGF population after conception, and the subsequent decline until age at menopause. The figure shows the dataset $(n=325)$, the model, the $95 \%$ prediction limits of the model, and the $95 \%$ confidence interval for the model. The horizontal axis denotes age in months up to birth at age zero, and age in years from birth to 51 years. Reprinted with permission from Wallace WH and Kelsey TW. PLoS One 2010; 5: e8772 [10].

\subsection{Mechanisms of radiation and chemotherapy induced damage}

Cytotoxic chemotherapy and radiotherapy can cause a reduction in the number of ovarian follicles. Both specifically target dividing cells, thus growing follicles are important sites of damage, but primordial follicles may also be lost through either a direct effect, or indirectly perhaps through increased initiation of growth [27] (figure 2).

\subsection{Impact of radiotherapy}

Modelling studies based on modern radiotherapy planning have demonstrated that the dose required to destroy $50 \%$ of non-growing follicles $\left(\mathrm{LD}_{50}\right)$ is $2 \mathrm{~Gy}$ [28]. Younger patients have higher residual follicle densities hence are more protected against POI from radiation, compared with adolescents and adults $[28,29]$. Prepubertal patients are at high risk of POI 
when exposed to ovarian radiation $\geq 10-15 \mathrm{~Gy}$, with an estimated sterilising dose of approximately $20 \mathrm{~Gy}[2,28]$. However a recent report from the St Jude Lifetime Cohort of childhood cancer survivors (median age 31.7 years), demonstrated that POI could be associated with any dose of ovarian radiation $[<1 \mathrm{~Gy}(\mathrm{HR}=13.85)$ or $\geq 1 \mathrm{~Gy}(\mathrm{HR}=132.34)][30]$. Post-pubertal patients are at high risk after exposure to $\geq 5-10 G y$, with a sterilising dose of approximately 16 Gy [28].

Radiation can also impact reproductive function through effects on the uterus, causing microvascular injury, endothelial damage and myometrial fibrosis resulting in poor uterine growth and distensibility $[29,31]$, resulting in miscarriage, preterm labour, low birth weight and stillbirth [32, 33, 34]. In contrast to the impact on ovarian function, the reproductive impact of uterine radiation is greater at a younger age, and pregnancy is of very high risk in those who have been exposed to uterine doses $>45$ Gy as adults or $>25 \mathrm{~Gy}$ in childhood [29].

\subsection{Impact of chemotherapy}

Ovarian damage from chemotherapy can occur via several mechanisms:

1. Direct DNA damage to growing ovarian follicles (via DNA breaks and inter-strand crosslinking) causes apoptosis [35]. Human xenografting models demonstrate that for high risk therapies such as cyclophosphamide, direct follicle loss can occur rapidly and drastically (53\% loss after 24 hours of the first dose) [35]. Studies of premenopausal women demonstrate an acute and rapid fall in $\mathrm{AMH}$ levels during chemotherapy, suggesting a similar rapid loss of growing follicles even in the absence of alkylators [36, 37, 38, 39]. Alkylators do not require cell proliferation for their cytotoxic action, thus they may also directly damage non-growing primordial follicles. Some follicles may survive through DNA repair but those that cannot are irretrievably lost [40]. 
2. The destruction of growing follicles and thus local reduction in $\mathrm{AMH}$ concentrations causes an upregulation in the PI3K/PTEN/Akt signalling pathway and loss of suppression of growth activation in primordial follicles $[41,42]$. Waves of primordial follicles become activated and excessively recruited into growing follicles which are more susceptible to the direct impact of chemotherapy, resulting in follicle burnout.

3. Direct damage to the ovarian stroma can result in fibrosis and hyalinisation of small blood vessels, resulting in indirect follicle damage and probably compromising growth [43, 44].

4. Both direct and indirect damage may alter the development of growing follicles and impair steroidogenesis, explaining the occurrence of temporary acute ovarian failure even with less ovo-toxic therapies [35]. As follicle growth takes many weeks, loss of the growing follicle population will result in amenorrhoea. Recovery will occur if sufficient primordial follicles remain to re-establish the growing follicle populations, with associated restoration of endocrine function and menstruation, discussed below.

\subsection{Clinical impact of gonadotoxic treatment on ovarian function}

Chemotherapy induced amenorrhoea due to acute ovarian failure is a common occurrence, however many women experience later recovery of ovarian function: this is very closely associated with age, as younger women have a greater ovarian reserve and thus are more likely to have sufficient follicle numbers after treatment $[45,46]$. The most significant long term consequences are POI (menopause by 40 years of age) and infertility (figure 2). POI may be immediate and permanent if the primordial follicle pool is completely depleted, or it may occur years after gonadotoxic treatment if there is only partial loss of primordial follicles which can then sustain ovarian function for a variable period (figure 3). As well as 
impact on fertility, $\mathrm{POI}$ is associated with chronic health sequelae including reduced life expectancy due to cardiovascular disease [47]; reduced bone mineral density (BMD), low muscle mass [30], psychosexual dysfunction [48]; genitourinary symptoms [49]; and possible cognitive impairment [50]. Women with POI have a 5-10\% chance of spontaneous conception, and should use contraception if they wish to avoid pregnancy [51]. Those who do conceive after chemotherapy show no increase in obstetric or neonatal risk, including congenital malformation or miscarriage $[52,53]$.

Large cohort studies of childhood cancer survivors have demonstrated the risk of acute ovarian failure by 5 years is $6.3 \%$ [54], of $\mathrm{POI}$ is $10.9 \%-32 \%[30,55]$, and of infertility is $16 \%$, [56]. In adults, there are much less data on fertility in large cohorts, with most studies reporting surrogates such as ongoing menses or ovarian reserve markers. The prevalence of early menopause has also been reported in a large survey of adult Hodgkin lymphoma survivors, highlighting the range of risk with different treatments, from a hazard ratio of 0.7 for ABVD (adriamycin, bleomycin, vinblastine and dacarbazine) to 35.6 for pelvic radiotherapy with alkylating agent chemotherapy [57]. The available data reflect the wide range of ages of women included and different treatment regimens. Thus in breast cancer patients chemotherapy induced amenorrhoea has been reported in $16-98 \%$ of women by the end of their treatment, with around $50 \%$ recovery in menses by two years, and a further $8 \%$ recovery up to 5 years $[46,58,59]$.

Individual risk of POI can be classified as low (<20\%), medium $(20-80 \%)$ and high $(>80 \%)$, and according to age, agent and dose of treatment [60]. Treatments considered low risk are antimetabolites (such as methotrexate, cytarabine) and vinca alkaloids (vincristine, vinblastine) which do not cause damage to human follicles. Anthracycline antibiotics are 
considered low risk, apart from Adriamycin which along with platins are considered 'medium risk'. Agents considered 'high-risk' for infertility include pelvic radiation; alkylators (such as cyclophosphamide $\geq 7.5 \mathrm{~g} / \mathrm{m}^{2}$, busulfan $\geq 600 \mathrm{mg} / \mathrm{m}^{2}$, ifosfamide $\geq 60 \mathrm{~g} / \mathrm{m}^{2}$, combination alkylating agent treatment); triazenes (such as procarbazine, $\geq 3$ cycles of MOPP [mustargen, oncovin, procarbazine, prednisone]); nitrosoureas (such as lomustine); combination alkylating agent treatment and ovarian radiotherapy $(\mathrm{HR}=95.56)[30,61]$. Myeloablative conditioning for stem cell transplantation (with high dose alkylating agents and total body irradiation) is associated with $65-95 \%$ risk of infertility in adult women [62]. The outcome is different for prepubertal girls who, because of higher ovarian reserve, have a risk of future gonadal insufficiency of around 50\% after stem cell transplantation [63]. Recent reports show that childhood survivors who received alkylators alone without radiation (with the exception of busulfan or lomustine), have either similar fertility to controls [64] or reduced chance of livebirth only at the highest quartile cyclophosphamide equivalent dose $\left(\geq 11.3 \mathrm{~g} / \mathrm{m}^{2}\right)$ [HR $\left.\left.0.85,0.74-0.98\right]\right)$ [61]. However the reduced fertility with delayed childbearing may be accentuated in cancer survivors $[61,64]$, and measures of ovarian reserve may be impaired in survivors with regular menstrual cycles $[39,65]$. Therefore, there is no clear accepted threshold for a "safe" alkylating agent dose with respect to future reproductive function [2]. 


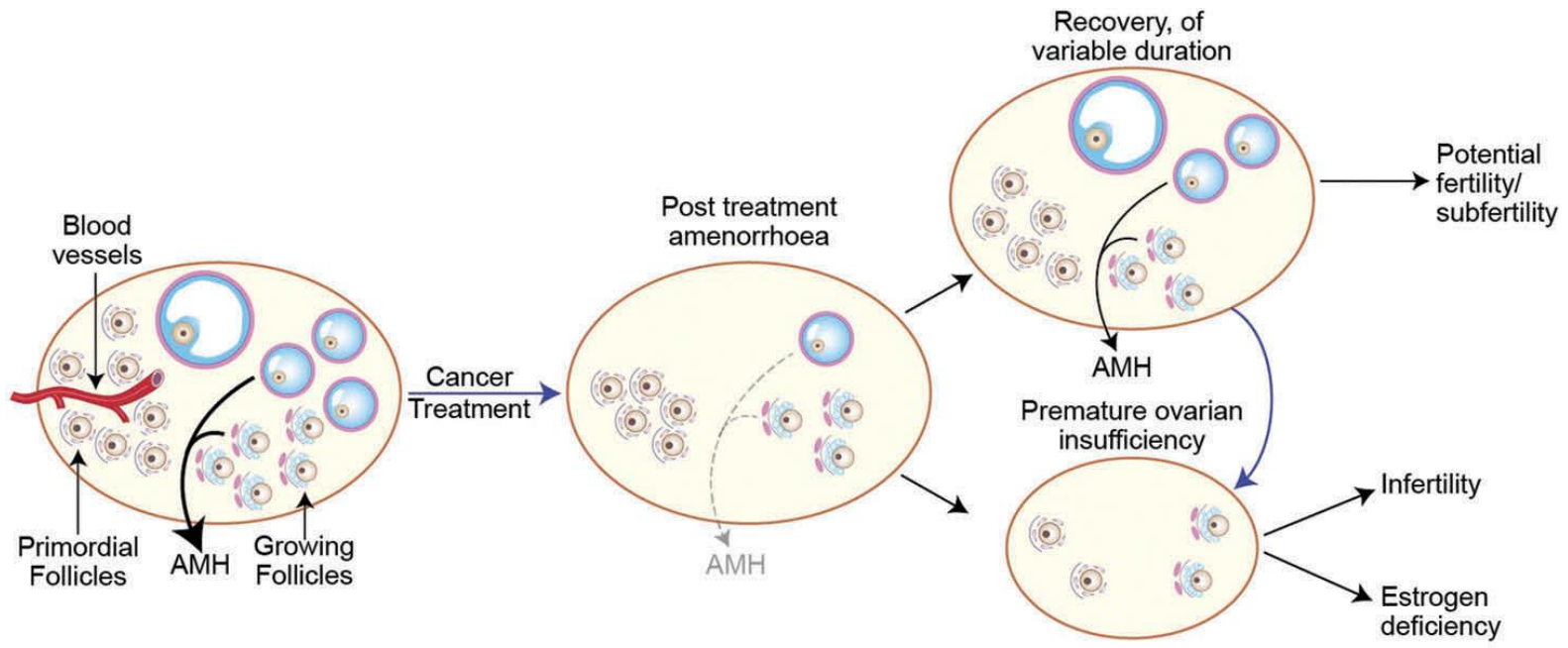

Figure 2. Representation of the effects of gonadotoxic treatment on the ovary. The number of primordial and early growing follicles in a healthy ovary is reduced by some chemotherapy regimens, with additional effects on the ovarian vasculature and stroma. If a sufficient population of primordial and thus early growing follicles remains, development of preovulatory follicles will continue allowing the potential for post-treatment fertility. Otherwise, complete depletion results in POI, infertility and estrogen deficiency. Ongoing posttreatment ovarian function may develop into $\mathrm{POI}$, depending on the remaining ovarian reserve.

\section{$\underline{4.1 \text { Fertoprotective Cancer treatments and gonadal protectants }}$}

New molecular targeted agents (such as monoclonal antibodies, kinase inhibitors, immunomodulators including melatonin), are in various stages of investigation, however their impact on ovarian function is largely unknown $[66,67,68]$. There have been reports proposing a likely transient effect of bevacizumab (an anti-VEGF agent) on ovarian function [69]. Other agents may have as yet unknown effects on the non-growing primordial follicle pool through activity on physiological pathways that are involved in the control of follicle dormancy and follicle growth activation. One example of this is imatinib, a tyrosine kinase inhibitor which has an important role in the treatment of chronic myeloid leukaemia, and has been reported to have adverse effects on ovarian function [70], but may also have protective effects as shown in an in-vitro model against the gonadotoxicity of cisplatin [71].

There is a lot of interest around Gonadotropin releasing hormone agonists ( $\mathrm{GnRHa}$ ) as a gonadal protectant during chemotherapy. GnRHa initially stimulates FSH and LH then causes suppression of hypothalamic-pituitary-ovarian axis by desensitisation of pituitary GnRH 
receptors. GnRHa may potentially protect ovarian reserve by keeping non-growing follicles in a dormant state, or by reducing ovarian perfusion and gonadotoxic penetration to follicles. Seven meta-analyses have been undertaken since 2011, the latest including 12 RCTs of 1231 breast cancer patients [72]. They demonstrate a decreased rate of POI (OR 0.36-0.55), increased resumption of menses (OR 1.90-3.46), and spontaneous ovulation (OR 2.70-5.70) in those receiving GnRHa cotreatment with conventional chemotherapy. Improved pregnancy rates with GnRHa cotreatment has also been suggested (OR 1.83) [72] although this is difficult to interpret without data on the number of women who tried to conceive. The two most recent and largest RCTs demonstrate benefit of GnRHa administration. The POEMS trial $(n=218)$ demonstrated that $\mathrm{GnRHa}$ reduced the risk of POI ( $8 \%$ versus $22 \%, H R \quad 0.30, p=0.04)$ ) (defined as amenorrhoea at 2 years for at least 6 months and elevated $\mathrm{FSH}$ ) and increased chance of pregnancy ( $22 \%$ versus $11 \%, \mathrm{p}=0.03)$ in women with operable hormone receptor negative breast cancer [73]. The GnRHa group also demonstrated improved disease-free survival. Similarly the OPTION trial in women with up to stage IIB breast cancer (receptor positive or negative) demonstrated a reduced risk of POI ( $2.6 \%$ versus $20.0 \%$ ) and amenorrhoea ( $10 \%$ versus $25.4 \%$ ) but only in women $\leq 40$ years [74]. However AMH levels did not significantly differ between groups suggesting that any protection of ovarian reserve is small, and this approach should not be used in place of more established fertility preservation methods, such as oocyte or embryo cryopreservation, where required. Additionally, there are no clear supportive data in other diagnoses: in lymphoma, the first long-term RCT (with 5 -6 year follow-up post GnRHa), reported no improvements in $\mathrm{POI}, \mathrm{AMH}$ or pregnancy rates with GnRHa co-treatment although the study was small compared to those in breast cancer, and there was substantial loss to follow-up [75]. 
As oncology paradigms move towards quality of life issues beyond cancer, fertoprotective agents will likely become available for clinical use, but much work needs to be done to assess their full impact on efficacy of cancer treatment and protection of fertility [76].

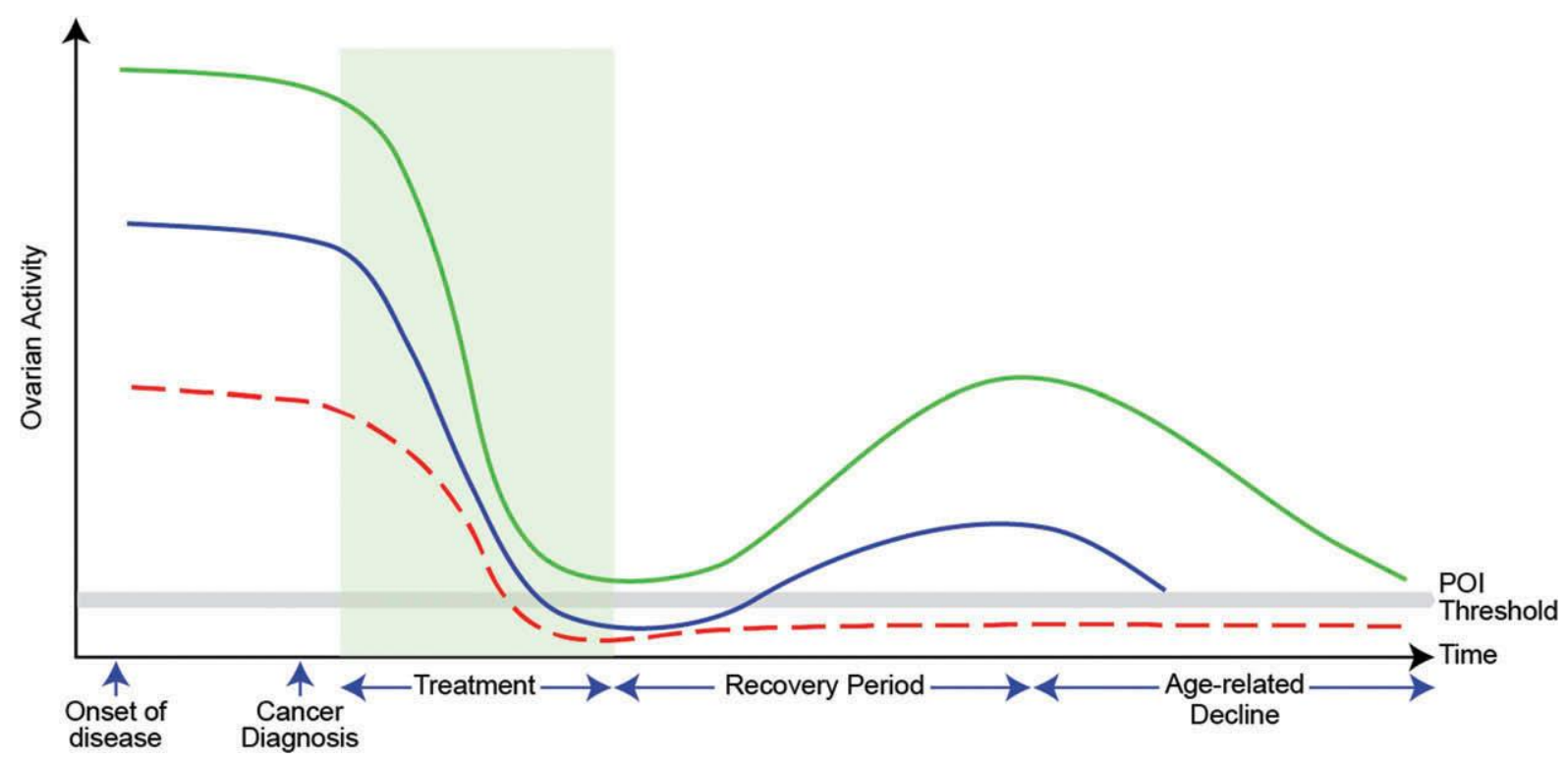

Figure 3. Schematic of the effect of gonadotoxic cancer treatment on ovarian function. The 3 lines represent women with high, average and low ovarian reserve (as e.g. reflected in AMH concentrations). Treatment results in a rapid fall in all women. Those with low ovarian reserve (red line) are more likely to develop POI during treatment, and for that to persist thereafter. Conversely, those with higher ovarian reserve will show a variable recovery, some going on to develop early POI (blue line) with others, at the highest level of ovarian reserve (green line) showing more prolonged ovarian activity, with later the normal age related decline.

\subsection{Surveillance of ovarian function}

\section{$\underline{5.1 \text { Indications }}$}

Currently ovarian function surveillance (involving evaluation of clinical biochemical and ultrasonographic markers of ovarian function) is an important aspect of care prior to gonadotoxic treatment and during survivorship. There are three main indications for surveillance:

1. To diagnose ovarian decline or established ovarian failure. The diagnosis of POI rests on the presence of (i) amenorrhoea for at least 4 months, and (ii) elevated follicle stimulating hormone $(\mathrm{FSH})>25 \mathrm{IU} / \mathrm{L}$ on two occasions $>4$ weeks apart [51]. POI needs to be 
excluded in those with pubertal delay or arrest, menstrual cycle dysfunction or menopausal symptoms [2]. It is recommended that sex steroid medications be discontinued for 2 months prior to testing, with care to avoid pregnancy [2].

2. To inform women of their reproductive prospects and guide decisions around assisted reproduction and fertility preservation. Once POI is established or imminent, the opportunity for biological parenthood is largely missed, as the chance of natural conception becomes significantly reduced about a decade beforehand [77]. A period of regular menstrual cyclicity can still occur in the presence of significant follicle depletion and reduced reproductive capacity $[36,78]$. Therefore postpubertal females who desire assessment regarding future fertility should be offered ovarian reserve testing. Low ovarian reserve may indicate a reduced reproductive lifespan, but does not reflect ability to conceive in the short term $[8,79]$, which is more determined by factors including age, regular ovulation and of course the partner's fertility. Additionally, while AMH predicts the ovarian response to stimulation, it is a poor predictor of live birth from assisted reproduction [80].

3. A potential future indication is to assess ovarian status after breast cancer treatment and provide guidance on choice of endocrine therapy [24]. Approximately $30 \%$ of women are premenopausal at diagnosis of breast cancer [81]. Adjuvant chemotherapy improves disease-free and overall survival [82] and has evolved from alkylating-based treatments (cyclophosphamide, methotrexate,5-fluorouracil (CMF)), to anthracycline or taxane based regimens. Endocrine therapy is an important systemic treatment for hormone receptor positive cancer, as ovarian suppression or oestrogen blockade reduces risk of recurrence. Tamoxifen (plus or minus the use of a GnRH analogue) is recommended as adjuvant treatment for premenopausal women [83], with aromatase inhibitors recommended in postmenopausal women [84]. Aromatase inhibitors block extraovarian oestrogen 
production, and reduce circulating oestrogen by $98 \%$ in postmenopausal women. They are contraindicated in perimenopausal and premenopausal women with endogenous oestrogen production, as oestrogen blockage may increase FSH leading to a paradoxical rise in ovarian steroid production. Chemotherapy induced amenorrhoea does not predict postmenopausal status as up to $32 \%$ of women $\geq 40$ years may recover ovarian function $[85,86]$. There is therefore a need for more sensitive markers of ovarian function that could more accurately predict likelihood of ovarian recovery in these patients [24].

\subsection{Candidate biomarkers:}

There are a number of candidate biomarkers of ovarian function including FSH, inhibin B, $\mathrm{AMH}$, and transvaginal ultrasound to assess antral follicle count (AFC, the total number of antral follicles [2-10mm] on both ovaries via transvaginal ultrasound on day 2-4 of the cycle), and ovarian volume. During gonadotoxic treatment, follicle depletion results in acute falls in circulating $\mathrm{AMH}$ and inhibin $\mathrm{B}, \mathrm{AFC}$ and ovarian volume, and an acute rise in FSH [37, 39] (figure 3). Recovery of these markers after cessation of therapy reflects reinitiation of follicle growth [39]. Thus, they are all in fact biomarkers of growing follicles (the "functional" ovarian reserve) [87], and only indirectly reflect the size of the primordial follicle pool (the "true" ovarian reserve).

Each of these markers has variable utility. Elevated FSH and reduced inhibin B are correlated with low ovarian reserve [88]; however normal levels may also be seen, and there may be significant variability in levels between and within menstrual cycles [3]. Ovarian volume increases from birth, is maximal after puberty, and declines with age until the menopause [89]. There is a strong positive correlation between ovarian volume and ovarian 
reserve [90] which has enabled development of predictive models of follicle density (the number of non- growing follicles per cubic millimetre of ovarian cortex) for healthy adult women [91]. There are significant correlations between ovarian volume, AFC, high gonadotrophins and amenorrhea due to gonadotoxic therapy [39, 78, 92]. Ultrasound measures however show significant intra and inter-operator variability [93].

$\mathrm{AMH}$ is currently the most robust marker of ovarian reserve in females over 25 years_[80, 93, 94, 95]. AMH levels are more discriminatory than $\mathrm{FSH}$, inhibin $\mathrm{B}, \mathrm{AFC}$ and ovarian volume $[24,36,37,88,93,96]$. They show little clinically significant fluctuation with menstrual cycles, or time of day $[97,98,99]$, although there remains an issue with assay standardisation, despite new automated assays becoming available [93]. For any given age levels vary substantially between individuals $[20,21]$. Both $\mathrm{AMH}$ and antral follicle count are reduced by use of hormonal contraception and $\mathrm{GnRH}[37,100]$. AMH levels can also vary with ethnicity and comorbidities (for example, being elevated in polycystic ovary syndrome, and reduced in women with lymphoma and in BRCA1 carriers) $[93,101,102,103]$. $\mathrm{AMH}$ levels predict time to menopause in older healthy women [104]. This may distinguish women who are at risk of POI but still have a reasonable follicle pool [105]. Predictive value is less accurate in younger women, with rate of decline of $\mathrm{AMH}$ being potentially a more significant factor in the latter group [96]. There are limited data to show an association between $\mathrm{AMH}$ and fecundity, conception or live birth [8],[80, 94],[106]. AMH is often measured prior to IVF, as low levels predict a poor oocyte yield in response to ovarian stimulation $[103,107,108]$.

$\mathrm{AMH}$ is an accurate marker of ovarian reserve after gonadotoxic treatment. AMH levels remain low to undetectable at completion of treatment, or show recovery depending on 
age and the drugs used $[24,39,78,109,110,111]$. In studies using less sensitive assays, low $\mathrm{AMH}$ was associated with amenorrhoea, however $\mathrm{AMH}$ was undetectable in some women with ongoing menses $[78,111]$. Highly sensitive and automated assays are now available which provide a more accurate assessment of ovarian function, and potential for recovery post chemotherapy. Undetectable AMH on ultrasensitive assay at completion of chemotherapy (where the limit of detection of AMH was $0.07 \mathrm{pmol} / \mathrm{l}[0.010 \mathrm{ng} / \mathrm{ml}]$ ), predicted POI at 24 months with good sensitivity and specificity (0.91 and 0.82 respectively) [24]. Women with detectable AMH at 2 years post treatment, were likely to have ongoing menses for at least 3 years, while those with an undetectable $\mathrm{AMH}$ at 2 years were considered to have no remaining ovarian reserve [88]. Additionally, improved assay sensitivity can allow detection of ovarian function after ovarian tissue reimplantation, and correlates with the resumption of ovulation in such patients [112].

There are limited data on the interpretation of $\mathrm{AMH}$ in girls and adolescents. Healthy children with low AMH still progress through normal puberty, although fertility data are lacking [21]. Small prospective studies have demonstrated a similar rapid decline in AMH with gonadotoxic treatment in children and adolescents as seen in adults $[113,114]$. Recovery of $\mathrm{AMH}$ was less likely in those having high risk treatment [114]. Low AMH was associated with absent pubertal development and menses in survivors [115], indicating that it is likely to be of value in early detection of those young girls who will not go through puberty naturally, allowing timely initiation of treatment.

\subsection{Pretreatment predictors and factors determining recovery of ovarian function:}

It would be very useful to predict the impact of cancer treatment on ovarian function prior to commencement. The pattern of ovarian recovery varies significantly according to a 
number of intrinsic and extrinsic factors, most importantly age and chemotherapy regimen. Development of personalised ovarian function scores, using a combination of multiple intrinsic and extrinsic factors, may provide more precise estimates of ovarian function and allow women to make informed decisions about the nature of their cancer treatment, and fertility care $[36,116,117,118]$.

\subsection{Intrinsic Factors determining recovery of ovarian function}

1. Advanced reproductive age: Age is the most important factor determining prevalence and duration of chemotherapy induced amenorrhoea, and recovery of ovarian reserve markers $[46,58,117]$. In breast cancer patients, around $70 \%$ of women under 40 years of age experience amenorrhoea, however prevalence and recovery of amenorrhoea is age dependent $[46,117,119]$. A two-year follow-up study of patients treated with anthracycline-based chemotherapy, demonstrated that $8.7 \%$ of the patients $<40$ years were still amenorrhoeic, compared to $85.6 \%$ of patients $\geq 40$ years [HR 12.26 (95\% Cl $5.21-28.86$ )] [46]. Ruddy et al. found that for every 1 year increase in age, there was an $18 \%$ increase in the odds of developing chemotherapy related amenorrhoea at 18 months [120].

2. Underlying ovarian reserve: Many studies assessing predictive value of ovarian reserve markers are reported on early breast cancer survivors so generalisability to other conditions is unclear. Several studies have demonstrated that pretreatment AMH is a good predictor of return of menses from $1-5$ years post treatment $[24,36,39,78,117,118,120$, 121] . Anderson et al. reported a $98.2 \%$ sensitivity, and $80.0 \%$ specificity for recovery of menses using a classification mosaic (figure 4) based on pretreatment age (greater than or less than 38.6 years) and AMH (below $3.8 \mathrm{pmol} / \mathrm{L}$ predicted amenorrhoea, above 20.3 pmol/L predicted ongoing menses) [36]. D’Avila et al. suggested that patients aged over 32 
years have counselling for fertility preservation prior to chemotherapy when pretreatment AMH levels are $<3.32 \mathrm{ng} / \mathrm{mL}(23.7 \mathrm{pmol} / \mathrm{L})$ [117]. Similar relationships between FSH, inhibin and ovarian recovery are not consistently seen $[36,118,122]$. A baseline AFC $<8$ or 9 was predictive of amenorrhoea post therapy $[78,117]$ while AFC $>19$ predicted ovarian function post therapy [78].

3. Other protective factors: larger body size, caucasian ethnicity, and genetic polymorphisms may protect ovarian function, although results are inconsistent $[2,86,118]$.

\subsection{Extrinsic factors influencing ovarian function recovery}

1. Smoking: In a study of premenopausal breast cancer patients, smoking was associated with lower estrogen levels, smaller ovaries, a lower AFC and amenorrhoea two years after chemotherapy compared to non-smoking [92]. Nonsmokers were 13 times more likely to recover menses.

2. High versus low risk gonadotoxic treatment: ovarian recovery varies significantly according to protocol [123]. Women who do not receive alkylating treatment have significantly faster recovery of AMH levels (even within 6 months of treatment cessation) and have higher $\mathrm{AMH}$, inhibin, estradiol levels, and a higher AFC in survivorship compared to those who receive alkylating treatment $[36,38,39,111]$. This may be due to the direct impact of alkylators on the primordial follicle pool, which is less likely in non-alkylators. Similar results have been seen in childhood and adolescent cancer survivors with those having low risk treatment demonstrating recovery of AMH by around 6 months as opposed to little or no recovery in those receiving high risk treatments (according to agent, dose, and ovarian radiation exposure) [114]. 


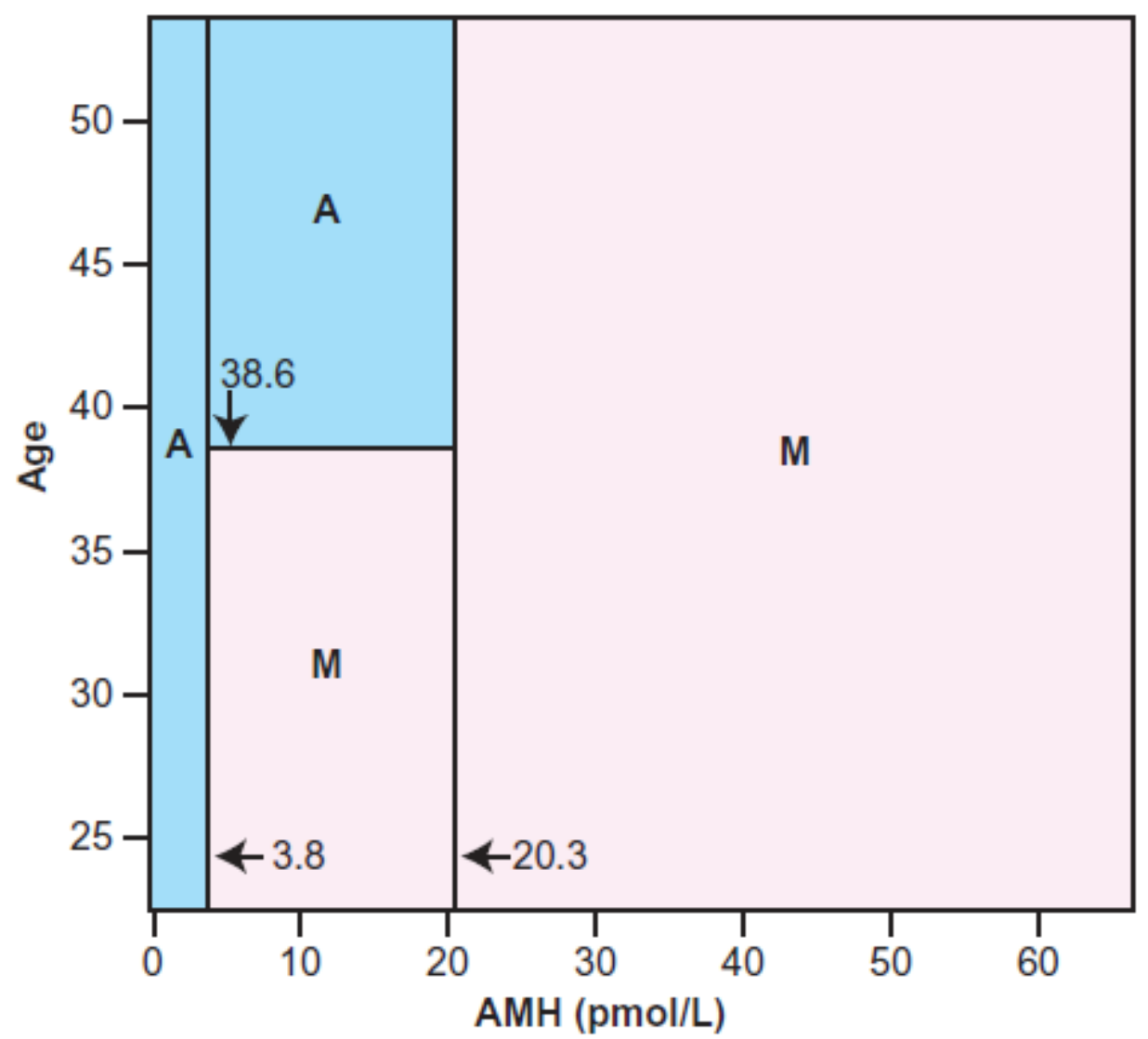

Figure 4. Prognostic mosaic chart using age and AMH to predict menses (M) or amenorrhoea (A) at 2-5 years post-treatment in women with early breast cancer (reproduced with permission from Anderson RA, et al. Eur J Cancer 2013; 49: 3404-3411) [36].

\subsection{Expert commentary}

The ovary is a very dynamic organ whose growing follicles are very sensitive to chemotherapy. Post-treatment function is however largely based on the survival of primordial follicles, which are impossible to quantify at present, and the best currently available biomarkers reflect activity of growing follicles some distance in time from their non-growing state. Non-invasive assessment of the ovarian reserve is thus the ultimate goal in this field. Clinical assessment at present does not allow accurate prediction of ongoing ovarian function (i.e. time to menopause), nor can it predict the ability to conceive. Another key gap in clinical management is that ovarian reserve testing cannot provide patients with individualised pretreatment assessment of infertility risk. Large cohort studies are available 
in childhood cancer survivors regarding fertility risk with analysis of specific drug classes and doses, as well as the impact of radiotherapy, but these are lacking in adult patients in whom largely short term outcomes having been reported (such as menses and using biomarkers). Thus patients are unable to make fully informed individualised risk-benefit decisions regarding their future fertility. To address this, long term follow-up studies in adults focussing on fertility as the key outcome (in addition to age at menopause/POI) are required. At all ages the distinctions between ovarian activity, fertility, and the desire and ability to achieve a pregnancy are important, and studies assessing later fertility have not distinguished between women unable to have a pregnancy and those who did not wish to become pregnant. This is particularly important in intervention studies such as those investigating ovarian protection strategies.

The last two decades have however seen very substantial increases in our understanding of the effects of cancer therapies on the ovary, albeit using endocrine rather than fertility outcomes. There are several approaches to ovarian protection in development, mostly preclinical at present, which offer potential. The possibility of constructing artificial ovaries has potential both for research and treatment: the complex pattern of follicle development and its long duration complicate in vitro replication, as well as the difficulties of using human tissue in such research. Rodent models have limitations in replicating human outcomes, and the ovaries of large animals such as sheep and cows are used disappointingly infrequently although are much closer to the human in structure and function. The long initial period of gonadotropin-independant folliculogenesis from initiation of follicle growth is also poorly understood, yet these follicles with their high proliferative activity are very sensitive to chemotherapy, and their number and activity is increasingly recognised to be of 
critical importance in the maintenance of primordial follicle growth arrest and its regulated activation.

The relatively quiescent nature of gonadal activity before puberty and the recognition that ovarian function continues to develop for many years after puberty are a barrier to the development of accurate tests and biomarkers for girls and adolescents. Although AMH is detectable in girls of all ages, its interpretation as a marker of ovarian reserve in children, adolescents and young women is very unclear and currently there are no evidence-based tests available for clinical use. This means that the management of hypogonadism in young people is generally instigated when there are clinical manifestations of ovarian dysfunction such as delayed growth and puberty, oligomenorrhoea or POI: the validation of $\mathrm{AMH}$ or other biomarkers in this context would be of considerable clinical value.

The analysis of ovarian function during and after cancer treatment is a burgeoning and rapidly advancing area of research, and as our knowledge of follicular development expands, our ability to counsel and manage patients with greater precision will improve.

\subsection{Five-year view}

Precision medicine will allow more accurate clinical prediction of ovarian failure, based on algorithms which combine a range of biomarkers and clinical factors to provide a personalised risk score. Ovarian function scores for premenopausal breast cancer patients have been reported in small studies, using various combinations of age, FSH, estradiol, $\mathrm{AMH}, \mathrm{BMI}$, and $\mathrm{AFC}[36,116,117,118]$. Combined analysis of these (and perhaps other) markers will improve the accuracy of prediction of return of ovarian function and prediction 
of remaining reproductive lifespan, but they are as yet inadequately validated for clinical use. These can be developed for prepubertal girls as well as for adult women. The development of a non-invasive method for assessment of the size of the ovarian reserve remains a fundamental need to allow more accurate assessment of ovarian function and the impact of gonadotoxic therapy. There also remains no method of predicting female fertility, in the absence of POI. At present, a woman's age is the only indicator we have of oocyte quality, which becomes the limiting factor in older women. This is a pressing need for reproductive medicine in general, particularly with the ongoing increase of the age at which women are having children, as they negotiate the competing demands of parenthood with education and financial stability $[124,125]$. These factors may have even greater impact for women after cancer treatment.

\section{Key issues}

- Significant improvements in cancer survival mean that ovarian function and fertility are important survivorship considerations.

- Assessing post-treatment ovarian function is important for young survivors.

- This may allow counselling regarding reproductive lifespan, and detection and timely treatment of POI.

- Prediction of post-treatment ovarian function before treatment starts may inform decisions regarding fertility preservation.

- The interaction of age and ovarian reserve markers is insufficiently understood at present. 
- Ultrasensitive AMH assays are a good marker of ovarian reserve in adults but do not predict short-term fertility.

- Emerging fertoprotective therapies require ongoing evaluation to assess their impact on fertility and cancer treatment.

\section{Disclosures}

YJ is funded by a National Health and Medical Research Council Fellowship and the Victorian Cancer Agency. RAA's work in this field has been supported by MRC grants G1100357 and $\mathrm{MR} / \mathrm{N022556/1}$ 


\section{References}

1. Ferlay J, Soerjomataram I, Ervik M, et al. GLOBOCAN 2012 v1.0, Cancer Incidence and Mortality Worldwide: IARC CancerBase No. 11 [Internet]. Lyon, France: International Agency for Research on Cancer; 2013 [updated 2012; cited 2017 28th November]: Available from: http://globocan.iarc.fr,

2. van Dorp W, Mulder RL, Kremer LC, et al. Recommendations for premature ovarian insufficiency surveillance for female survivors of childhood, adolescent, and young adult cancer: A report From the International Late Effects of Childhood Cancer Guideline Harmonization Group in Collaboration With the PanCareSurFup Consortium. JCO 2016;34(28):3440-50.

3. Bedoschi G, Navarro PA, Oktay K. Chemotherapy-induced damage to ovary: mechanisms and clinical impact. Future Oncol 2016;12(20):2333-44.

4. Crawshaw M. Psychosocial oncofertility issues faced by adolescents and young adults over their lifetime: a review of the research. Hum Fertil (Camb) 2013 ;16(1):59-63.

5. Lehmann V, Keim MC, Nahata L, et al. Fertility-related knowledge and reproductive goals in childhood cancer survivors: short communication. Hum Reprod 2017 ;32(11):2250-2253.

6. Nilsson J, Jervaeus A, Lampic C, et al. 'Will I be able to have a baby?' Results from online focus group discussions with childhood cancer survivors in Sweden. Hum Reprod 2014;29(12):2704-11.

7. Letourneau JM, Ebbel EE, Katz PP, et al. Acute ovarian failure underestimates agespecific reproductive impairment for young women undergoing chemotherapy for cancer. Cancer 2012 ;118(7):1933-9. 
8. Steiner AZ, Pritchard D, Stanczyk FZ, et al. Association between biomarkers of ovarian reserve and infertility among older women of reproductive age. JAMA 2017 318(14):1367-1376.

* 8 year prospective cohort study of over 900 women aged $30-44$ years demonstrating that ovarian reserve markers did not predict ability to conceive

9. Findlay JK, Hutt KJ, Hickey M, et al. How is the number of primordial follicles in the ovarian reserve Established? Biol Reprod 2015 Nov;93(5):111.

10. Wallace WH, Kelsey TW. Human ovarian reserve from conception to the menopause. PloS one $2010 ; 5(1): e 8772$.

** The first model ovarian reserve from conception to menopause, which could estimate the number of non-growing follicles in the ovary at any given age.

11. Peters H, Byskov AG, Grinsted J. Follicular growth in fetal and prepubertal ovaries of humans and other primates. Clin Endocrinol Metab 1978;7(3):469-85.

12. Zuccotti M, Merico V, Cecconi S, et al. What does it take to make a developmentally competent mammalian egg? Hum Reprod Update 2011;17(4):525-40.

13. van Noord PA, Dubas JS, Dorland M, et al. Age at natural menopause in a populationbased screening cohort: the role of menarche, fecundity, and lifestyle factors. Fertil Steril $1997 ; 68(1): 95-102$.

14. Tucker EJ, Grover SR, Bachelot A, et al. Premature ovarian insufficiency: new perspectives on genetic cause and phenotypic spectrum. Endocr Rev 2016 ;37(6):609-635.

15. Kok HS, van Asselt KM, van der Schouw YT, et al. Genetic studies to identify genes underlying menopausal age. Hum Reprod Update 2005;11(5):483-93. 
16. Reddy $\mathrm{P}$, Zheng W, Liu K. Mechanisms maintaining the dormancy and survival of mammalian primordial follicles. Trends Endocrinol Metab 2010;21(2):96-103.

17. Durlinger AL, Gruijters MJ, Kramer $\mathrm{P}$, et al. Anti-Mullerian hormone inhibits initiation of primordial follicle growth in the mouse ovary. Endocrinology 2002;143(3):107684.

* An important study demonstrating the key role of $A M H$ in inhibition of folliculogenesis in the mouse ovary

18. Jeppesen JV, Anderson RA, Kelsey TW, et al. Which follicles make the most antimullerian hormone in humans? Evidence for an abrupt decline in $\mathrm{AMH}$ production at the time of follicle selection. Mol Hum Reprod 2013;19(8):519-27.

19. $\operatorname{de~Vet~A,~Laven~JS,~de~Jong~FH,~et~al.~Antimullerian~hormone~serum~levels:~a~putative~}$ marker for ovarian aging. Fertil Steril 2002;77(2):357-62.

20. Kelsey TW, Wright $P$, Nelson SM, et al. A validated model of serum anti-mullerian hormone from conception to menopause. PloS one 2011;6(7):e22024.

** The first model of circulating AMH levels from conception to menopause allowing normative data to be generated at all ages, and demonstrating an association between circulating levels and recruitment of non-growing follicles.

21. Hagen CP, Aksglaede L, Sorensen $\mathrm{K}$, et al. Individual serum levels of anti-mullerian hormone in healthy girls persist through childhood and adolescence: a longitudinal cohort study. Hum Reprod 2012;27(3):861-6.

22. Jeffery A, Streeter AJ, Hosking J, et al. Anti-mullerian hormone in children: a ten-year prospective longitudinal study (EarlyBird 39). J Pediatr Endocrinol Metab 2015;28(910):1153-62. 
23. Sowers MR, Eyvazzadeh AD, McConnell D, et al. Anti-mullerian hormone and inhibin $B$ in the definition of ovarian aging and the menopause transition. J Clin Endocrinol Metab 2008;93(9):3478-83.

24. Anderson RA, Mansi J, Coleman RE, et al. The utility of anti-Mullerian hormone in the diagnosis and prediction of loss of ovarian function following chemotherapy for early breast cancer. Eur J Cancer 2017 Dec;87:58-64.

** A study of breast cancer patients demonstrating that AMH levels measured on ultrasensitive assay at completion of chemotherapy could reliably predict presence or absence of menses 2 years after treatment.

25. Anderson RA, McLaughlin $M$, Wallace $W H$, et al. The immature human ovary shows loss of abnormal follicles and increasing follicle developmental competence through childhood and adolescence. Hum Reprod 2014;29(1):97-106.

26. Kano $M$, Sosulski $A E$, Zhang $L$, et al. $A M H / M I S$ as a contraceptive that protects the ovarian reserve during chemotherapy. Proc Natl Acad Sci U S A 2017;114(9):E1688E1697.

* A very interesting study demonstrating inhibition of folliculogenesis in mice after treatment with supraphysiological doses of $A M H$, indicating it may show promise as a gonadal protectant in the future.

27. Morgan S, Anderson RA, Gourley C, et al. How do chemotherapeutic agents damage the ovary? Hum Reprod Update 2012;18(5):525-35.

28. Wallace $\mathrm{WH}$, Thomson AB, Saran F, et al. Predicting age of ovarian failure after radiation to a field that includes the ovaries. Int J Radiat Oncol Biol Phys 2005 ;62(3):738-44. 
29. Teh WT, Stern C, Chander S, et al. The impact of uterine radiation on subsequent fertility and pregnancy outcomes. Biomed Res Int 2014;2014:482968.

30. Chemaitilly W, Li Z, Krasin MJ, et al. Premature ovarian insufficiency in childhood cancer Survivors: A report From the St. Jude Lifetime Cohort. J Clin Endocrinol Metab 2017;102(7):2242-2250.

31. Bath LE, Critchley HO, Chambers SE, et al. Ovarian and uterine characteristics after total body irradiation in childhood and adolescence: response to sex steroid replacement. Br J Obstet Gynaecol 1999;106(12):1265-72.

32. Signorello LB, Cohen SS, Bosetti C, et al. Female survivors of childhood cancer: preterm birth and low birth weight among their children. J Natl Cancer Inst 2006 ;98(20):1453-61.

33. Signorello LB, Mulvihill JJ, Green DM, et al. Stillbirth and neonatal death in relation to radiation exposure before conception: a retrospective cohort study. Lancet 2010 21;376(9741):624-30.

34. Salooja N, Szydlo RM, Socie G, et al. Pregnancy outcomes after peripheral blood or bone marrow transplantation: a retrospective survey. Lancet 2001;358(9278):271-6.

35. Oktem O, Oktay K. A novel ovarian xenografting model to characterize the impact of chemotherapy agents on human primordial follicle reserve. Cancer Res 2007 ;67(21):10159-62.

35. Anderson RA, Rosendahl M, Kelsey TW, et al. Pretreatment anti-mullerian hormone predicts for loss of ovarian function after chemotherapy for early breast cancer. Eur J Cancer 2013;49(16):3404-11.

${ }^{*} A$ study combining pretreatment $A M H$ levels and age that predicted with good sensitivity and specificity the likelihood of return of ovarian function after 
gonadotoxic treatment. A predictive classification mosaic was developed which could assist decision making regarding fertility preservation decisions prior to treatment.

37. Anderson RA, Themmen AP, Al-Qahtani A, et al. The effects of chemotherapy and long-term gonadotrophin suppression on the ovarian reserve in premenopausal women with breast cancer. Hum Reprod 2006;21(10):2583-92.

38. Rosendahl M, Andersen $\mathrm{CY}$, la Cour Freiesleben N, et al. Dynamics and mechanisms of chemotherapy-induced ovarian follicular depletion in women of fertile age. Fertil Steril 2010;94(1):156-66.

39. Dillon KE, Sammel MD, Prewitt M, et al. Pretreatment antimullerian hormone levels determine rate of post-therapy ovarian reserve recovery: acute changes in ovarian reserve during and after chemotherapy. Fertil Steril 2013;99(2):477-83.

40. Oktay K, Turan V, Titus S, et al. BRCA Mutations, DNA repair deficiency, and ovarian aging. Biol Reprod 2015;93(3):67.

41. Kalich-Philosoph L, Roness H, Carmely A, et al. Cyclophosphamide triggers follicle activation and "burnout"; AS101 prevents follicle loss and preserves fertility. Sci Transl Med $2013 ; 5(185): 185$ ra62.

42. Adhikari D, Liu K. Molecular mechanisms underlying the activation of mammalian primordial follicles. Endocr Rev 2009;30(5):438-64.

43. Soleimani R, Heytens E, Darzynkiewicz Z, et al. Mechanisms of chemotherapyinduced human ovarian aging: double strand DNA breaks and microvascular compromise. Aging (Albany NY) 2011;3(8):782-93. 
44. Meirow D, Dor J, Kaufman B, et al. Cortical fibrosis and blood-vessels damage in human ovaries exposed to chemotherapy. Potential mechanisms of ovarian injury. Hum Reprod 2007;22(6):1626-33.

45. Petrek JA, Naughton MJ, Case LD, et al. Incidence, time course, and determinants of menstrual bleeding after breast cancer treatment: a prospective study. JCO 2006 $1 ; 24(7): 1045-51$.

46. Vriens IJ, De Bie AJ, Aarts MJ, et al. The correlation of age with chemotherapyinduced ovarian function failure in breast cancer patients. Oncotarget 2017 ;8(7):11372-11379.

47. Wu X, Cai H, Kallianpur A, et al. Impact of premature ovarian failure on mortality and morbidity among Chinese women. PloS one 2014;9(3):e89597.

48. Marino JL, Saunders CM, Hickey M. Sexual inactivity in partnered female cancer survivors. Maturitas 2017;105:89-94.

49. Faubion SS, Sood R, Kapoor E. Genitourinary syndrome of menopause: management Strategies for the clinician. Mayo Clin Proc 2017 ;92(12):1842-1849.

50. Vearncombe KJ, Pachana NA. Is cognitive functioning detrimentally affected after early, induced menopause? Menopause 2009 ;16(1):188-98.

51. European Society for Human Reproduction, Embryology Guideline Group on POI, Webber L, et al. ESHRE Guideline: management of women with premature ovarian insufficiency. Hum Reprod 2016;31(5):926-37.

\section{* Consensus guidelines on management of POI}

52. Mueller BA, Chow EJ, Kamineni A, et al. Pregnancy outcomes in female childhood and adolescent cancer survivors: a linked cancer-birth registry analysis. Arch Pediatr Adolesc Med $2009 ; 163(10): 879-86$. 
53. Signorello LB, Mulvihill JJ, Green DM, et al. Congenital anomalies in the children of cancer survivors: a report from the childhood cancer survivor study. JCO 2012;30(3):239-45.

54. Chemaitilly W, Mertens AC, Mitby P, et al. Acute ovarian failure in the childhood cancer survivor study. J Clin Endocrinol Metab 2006;91(5):1723-8.

55. Hudson MM, Ness KK, Gurney JG, et al. Clinical ascertainment of health outcomes among adults treated for childhood cancer. JAMA 2013;309(22):2371-2381.

56. Barton SE, Najita JS, Ginsburg ES, et al. Infertility, infertility treatment, and achievement of pregnancy in female survivors of childhood cancer: a report from the Childhood Cancer Survivor Study cohort. Lancet Oncol 2013;14(9):873-81.

* Longterm follow up study of over 3500 female childhood cancer survivors from over 26 North American Institutions, demonstrating that survivors had an increased risk of infertility which was over 2.5 fold compared to sibling controls by 25 years of age.

57. Swerdlow AJ, Cooke R, Bates A, et al. Risk of premature menopause after treatment for Hodgkin's lymphoma. J Natl Cancer Inst 2014 ;106(9): doi: 10.1093/jnci/dju207.

* large survey demonstrating effects of different treatment regimens on risk of early menopause in Hodgkin lymphoma

58. Tham $\mathrm{YL}$, Sexton $\mathrm{K}$, Weiss $\mathrm{H}$, et al. The rates of chemotherapy-induced amenorrhea in patients treated with adjuvant doxorubicin and cyclophosphamide followed by a taxane. Am J Clin Oncol 2007;30(2):126-32. 
59. Okanami Y, Ito Y, Watanabe $C$, et al. Incidence of chemotherapy-induced amenorrhea in premenopausal patients with breast cancer following adjuvant anthracycline and taxane. Breast Cancer 2011;18(3):182-8.

60. Loren AW. Fertility issues in patients with hematologic malignancies. Hematology Am Soc Hematol Educ Program 2015;2015:138-45.

61. Chow EJ, Stratton KL, Leisenring WM, et al. Pregnancy after chemotherapy in male and female survivors of childhood cancer treated between 1970 and 1999: a report from the Childhood Cancer Survivor Study cohort. Lancet Oncol 2016;17(5):567-76.

** Longterm followup study of almost 11000 childhood cancer survivors compared to sibling controls showing that male surviviors had a high risk of infertility after exposure to alkylators, while female survivors were at risk after exposure to the highest quartile dose.

62. Tauchmanova L, Selleri C, De Rosa G, et al. Gonadal status in reproductive age women after haematopoietic stem cell transplantation for haematological malignancies. Hum Reprod 2003;18(7):1410-6.

63. Vatanen A, Wilhelmsson M, Borgstrom B, et al. Ovarian function after allogeneic hematopoietic stem cell transplantation in childhood and adolescence. Eur J Endocrinol $2014 ; 170(2): 211-8$

64. Bramswig JH, Riepenhausen M, Schellong G. Parenthood in adult female survivors treated for Hodgkin's lymphoma during childhood and adolescence: a prospective, longitudinal study. Lancet Oncol 2015;16(6):667-75.

** Analysis of chance of parenthood in a national population of female childhood Hodgkin lymphoma survivors, with analysis of treatment predictors of not achieving parenthood. 
65. Bath LE, Wallace WH, Shaw MP, et al. Depletion of ovarian reserve in young women after treatment for cancer in childhood: detection by anti-Mullerian hormone, inhibin B and ovarian ultrasound. Hum Reprod 2003;18(11):2368-74.

66. Wu KN, Luo Y, Liu LZ, et al. Twin pregnancy and childbirth after reduced-intensity conditioning allogeneic haematopoietic stem cell transplantation combined with imatinib mesylate for chronic myeloid leukaemia: case report and literature review. J Int Med Res 2012;40(6):2409-15.

67. Phelan R, Mann E, Napurski C, et al. Ovarian function after hematopoietic cell transplantation: a descriptive study following the use of GnRH agonists for myeloablative conditioning and observation only for reduced-intensity conditioning. Bone Marrow Transplant 2016 ;51(10):1369-1375.

68. Jang $\mathrm{H}$, Lee $\mathrm{OH}$, Lee $\mathrm{Y}$, et al. Melatonin prevents cisplatin-induced primordial follicle loss via suppression of PTEN/AKT/FOXO3a pathway activation in the mouse ovary. J Pineal Res $2016 ; 60(3): 336-47$.

69. Imai A, Ichigo S, Matsunami K, et al. Ovarian function following targeted antiangiogenic therapy with bevacizumab. Mol Clin Oncol 2017 ;6(6):807-810.

70. Zamah AM, Mauro MJ, Druker BJ, et al. Will imatinib compromise reproductive capacity? The Oncologist 2011;16(10):1422-7.

71. Gonfloni S, Di Tella L, Caldarola S, et al. Inhibition of the c-Abl-TAp63 pathway protects mouse oocytes from chemotherapy-induced death. Nature Medicine 2009 ;15(10):1179-85.

72. Lambertini $M$, Ceppi M, Poggio $F$, et al. Ovarian suppression using luteinizing hormone-releasing hormone agonists during chemotherapy to preserve ovarian 
function and fertility of breast cancer patients: a meta-analysis of randomized studies. Annal Oncol 2015;26(12):2408-19.

*A meta-analysis of 12 RCTs of GnRHa use demonstrating reduced rates of POI and improved pregnancy rates with GnRHa cotreatment

73. Moore HC, Unger JM, Phillips KA, et al. Goserelin for ovarian protection during breast-cancer adjuvant chemotherapy. NEJM 2015;372(10):923-32.

74. Leonard RCF, Adamson DJA, Bertelli G, et al. GnRH agonist for protection against ovarian toxicity during chemotherapy for early breast cancer: the Anglo Celtic Group OPTION trial. Annal Oncol 2017;28(8):1811-1816.

75. Demeestere I, Brice P, Peccatori FA, et al. No evidence for the benefit of gonadotropin-releasing hormone agonist in preserving ovarian function and fertility in lymphoma survivors treated with chemotherapy: final long-term report of a prospective randomized trial. JCO 2016;34(22):2568-74.

* The longest follow-up study after using GnRH cotreatment demonstrating that it did not preserve fertility in lymphoma survivors, at 5 years

76. Kim SY, Kim SK, Lee JR, et al. Toward precision medicine for preserving fertility in cancer patients: existing and emerging fertility preservation options for women. J Gynecol Oncol 2016;27(2):e22.

77. Tarlatzis BC, Zepiridis L. Perimenopausal conception. Ann N Y Acad Sci 2003 ;997:93104.

78. Anderson RA, Cameron DA. Pretreatment serum anti-mullerian hormone predicts long-term ovarian function and bone mass after chemotherapy for early breast cancer. J Clin Endocrinol Metabolism 2011;96(5):1336-43. 
79. Hagen CP, Vestergaard S, Juul A, et al. Low concentration of circulating antimullerian hormone is not predictive of reduced fecundability in young healthy women: a prospective cohort study. Fertil Steril 2012;98(6):1602-8 e2.

80. Iliodromiti S, Kelsey TW, Wu O, et al. The predictive accuracy of anti-Mullerian hormone for live birth after assisted conception: a systematic review and metaanalysis of the literature. Hum Reprod Update 2014;20(4):560-70.

\section{* A metanalysis demonstrating the limited accuracy of $A M H$ in predicting conception and live birth with assisted conception.}

81. Torino F, Barnabei A, De Vecchis L, et al. Chemotherapy-induced ovarian toxicity in patients affected by endocrine-responsive early breast cancer. Crit Rev Oncol Hematol 2014;89(1):27-42.

82. Early Breast Cancer Trialists' Collaborative Group. Effects of chemotherapy and hormonal therapy for early breast cancer on recurrence and 15-year survival: an overview of the randomised trials. Lancet 2005;365(9472):1687-717.

83. Regan MM, Francis PA, Pagani O, et al. Absolute benefit of adjuvant endocrine therapies for premenopausal womenwith hormone receptor-positive, human epidermal growth factor receptor 2-negative early breast cancer: TEXT and SOFT Trials. JCO 2016;34(19):2221-31.

84. Buzdar AU. Pharmacology and pharmacokinetics of the newer generation aromatase inhibitors. Clin Cancer Res 2003;9(1 Pt 2):468S-72S.

85. Guerrero A, Gavila J, Folkerd E, et al. Incidence and predictors of ovarian function recovery (OFR) in breast cancer (BC) patients with chemotherapy-induced amenorrhea (CIA) who switched from tamoxifen to exemestane. Annal Oncol 2013 ;24(3):674-9. 
86. Krekow LK, Hellerstedt BA, Collea RP, et al. Incidence and predictive factors for recovery of ovarian function in amenorrheic women in their 40s treated with letrozole. JCO 2016 10;34(14):1594-600.

87. Anderson RA, Nelson SM, Wallace WH. Measuring anti-mullerian hormone for the assessment of ovarian reserve: when and for whom is it indicated? Maturitas 2012 ;71(1):28-33.

88. Chai J, Howie AF, Cameron DA, et al. A highly-sensitive anti-mullerian hormone assay improves analysis of ovarian function following chemotherapy for early breast cancer. Eur J Cancer 2014;50(14):2367-74.

89. Kelsey TW, Dodwell SK, Wilkinson AG, et al. Ovarian volume throughout life: a validated normative model. PloS one 2013;8(9):e71465.

90. Kelsey TW, Wallace WH. Ovarian volume correlates strongly with the number of nongrowing follicles in the human ovary. Obstet Gynecol Int 2012;2012:305025.

91. McLaughlin M, Kelsey TW, Wallace WH, et al. An externally validated age-related model of mean follicle density in the cortex of the human ovary. J Assist Reprod Genet 2015;32(7):1089-95.

92. Wenners A, Grambach J, Koss J, et al. Reduced ovarian reserve in young early breast cancer patients: preliminary data from a prospective cohort trial. BMC Cancer 2017 $6 ; 17(1): 632$

93. Iliodromiti S, Anderson RA, Nelson SM. Technical and performance characteristics of anti-Mullerian hormone and antral follicle count as biomarkers of ovarian response. Hum Reprod Update 2015;21(6):698-710.

94. Broekmans FJ, Kwee J, Hendriks DJ, et al. A systematic review of tests predicting ovarian reserve and IVF outcome. Hum Reprod Update 2006;12(6):685-718. 
95. Broer SL, Dolleman M, Opmeer BC, et al. AMH and AFC as predictors of excessive response in controlled ovarian hyperstimulation: a meta-analysis. Hum Reprod Update $2011 ; 17(1): 46-54$

96. Freeman EW, Sammel MD, Lin $\mathrm{H}$, et al. Anti-mullerian hormone as a predictor of time to menopause in late reproductive age women. J Clin Endocrinol Metab 2012 ;97(5):1673-80.

97. La Marca A, Malmusi S, Giulini S, et al. Anti-Mullerian hormone plasma levels in spontaneous menstrual cycle and during treatment with FSH to induce ovulation. Hum Reprod. 2004;19(12):2738-41.

98. Kissell KA, Danaher MR, Schisterman EF, et al. Biological variability in serum antimullerian hormone throughout the menstrual cycle in ovulatory and sporadic anovulatory cycles in eumenorrheic women. Hum Reprod 2014;29(8):1764-72.

99. Bungum $L$, Jacobsson AK, Rosen $F$, et al. Circadian variation in concentration of antimullerian hormone in regularly menstruating females: relation to age, gonadotrophin and sex steroid levels. Hum Reprod 2011 ;26(3):678-84.

100. Johnson LN, Sammel MD, Dillon KE, et al. Antimullerian hormone and antral follicle count are lower in female cancer survivors and healthy women taking hormonal contraception. Fertil Steril 2014 Sep;102(3):774-781.

101. Phillips KA, Collins IM, Milne RL, et al. Anti-Mullerian hormone serum concentrations of women with germline BRCA1 or BRCA2 mutations. Hum Reprod 2016 ;31(5):112632.

* The largest series of women with BRCA1 and BRCA2 mutations, showing a reduction in AMH concentraitons in those with BRCA1 mutations 
102. Lauritsen MP, Bentzen JG, Pinborg A, et al. The prevalence of polycystic ovary syndrome in a normal population according to the Rotterdam criteria versus revised criteria including anti-Mullerian hormone. Hum Reprod 2014;29(4):791-801.

103. Lawrenz B, Fehm T, von Wolff M, et al. Reduced pretreatment ovarian reserve in premenopausal female patients with Hodgkin lymphoma or non-Hodgkinlymphoma--evaluation by using antimullerian hormone and retrieved oocytes. Fertil Steril $2012 ; 98(1): 141-4$.

104. Freeman EW, Sammel MD, Lin H, et al. Contribution of the rate of change of antimullerian hormone in estimating time to menopause for late reproductive-age women. Fertil Steril 2012;98(5):1254-9.

105. Visser JA, Schipper I, Laven JS, et al. Anti-Mullerian hormone: an ovarian reserve marker in primary ovarian insufficiency. Nat Rev Endocrinol 2012 ;8(6):331-41.

106. Brodin T, Hadziosmanovic N, Berglund L, et al. Antimullerian hormone levels are strongly associated with live-birth rates after assisted reproduction. J Clin Endocrinol Metab $2013 ; 98(3): 1107-14$

107. Arce JC, La Marca A, Mirner Klein B, et al. Antimullerian hormone in gonadotropin releasing-hormone antagonist cycles: prediction of ovarian response and cumulative treatment outcome in good-prognosis patients. Fertil Steril. 2013 ;99(6):1644-53.

108. Wu CH, Chen YC, Wu HH, et al. Serum anti-Mullerian hormone predicts ovarian response and cycle outcome in IVF patients. J Assist Reprod Genet 2009;26(7):383-9.

109. Su HI, Sammel MD, Green J, et al. Antimullerian hormone and inhibin B are hormone measures of ovarian function in late reproductive-aged breast cancer survivors. Cancer $2010 ; 116(3): 592-9$. 
110. Partridge $\mathrm{AH}$, Ruddy $\mathrm{KJ}$, Gelber $\mathrm{S}$, et al. Ovarian reserve in women who remain premenopausal after chemotherapy for early stage breast cancer. Fertil Steril 2010 ;94(2):638-44.

111. Decanter C, Morschhauser F, Pigny P, et al. Anti-mullerian hormone follow-up in young women treated by chemotherapy for lymphoma: preliminary results. Reprod Biomed Online 2010;20(2):280-5.

* Demonstration that AMH reveals differences in gonadotoxicity between 2 chemotherapy regimens

112. Dunlop CE, Brady BM, McLaughlin M, et al. Re-implantation of cryopreserved ovarian cortex resulting in restoration of ovarian function, natural conception and successful pregnancy after haematopoietic stem cell transplantation for Wilms tumour. J Assisted Reprod Genetics 2016 Dec;33(12):1615-1620.

113. Gupta AA, Lee Chong A, Deveault C, et al. Anti-mullerian hormone in female adolescent cancer patients before, during, and after completion of therapy: a pilot feasibility study. J Ped Adolesc Gynecol 2016;29(6):599-603.

114. Brougham MF, Crofton PM, Johnson EJ, et al. Anti-Mullerian hormone is a marker of gonadotoxicity in pre- and postpubertal girls treated for cancer: a prospective study. J Clin Endocrinol Metab 2012;97(6):2059-67.

115. Miyoshi $\mathrm{Y}$, Ohta $\mathrm{H}, \mathrm{Namba} \mathrm{N}$, et al. Low serum concentrations of anti-mullerian hormone are common in 53 female childhood cancer survivors. Horm Res Paediatr 2013;79(1):17-21.

116. Li XS, Lv Q, Du ZG, et al. Prediction of ovarian function in premenopausal breast cancer patients with amenorrhoea after chemotherapy: a simple clinical score. Springerplus. 2016;5(1):1052. 
117. D'Avila AM, Biolchi V, Capp E, et al. Age, anti-mullerian hormone, antral follicles count to predict amenorrhea or oligomenorrhea after chemotherapy with cyclophosphamide. J Ovarian Res 2015;14;8:82.

118. Su HC, Haunschild C, Chung K, et al. Prechemotherapy antimullerian hormone, age, and body size predict timing of return of ovarian function in young breast cancer patients. Cancer 2014 1;120(23):3691-8.

\section{* Analysis of predictors of recovery of ovarian function after chemotherapy}

119. Shapiro CL, Recht A. Side effects of adjuvant treatment of breast cancer. NEJM 2001 ;344(26):1997-2008.

120. Ruddy KJ, O'Neill A, Miller KD, et al. Biomarker prediction of chemotherapy-related amenorrhea in premenopausal women with breast cancer participating in E5103. Breast Cancer Res Treat 2014 ;144(3):591-7.

121. Henry NL, Xia R, Schott AF, et al. Prediction of postchemotherapy ovarian function using markers of ovarian reserve. The Oncologist 2014;19(1):68-74.

122. Anders C, Marcom PK, Peterson B, et al. A pilot study of predictive markers of chemotherapy-related amenorrhea among premenopausal women with early stage breast cancer. Cancer Invest 2008;26(3):286-95.

123. Peigne $M$, Decanter $\mathrm{C}$. Serum $\mathrm{AMH}$ level as a marker of acute and long-term effects of chemotherapy on the ovarian follicular content: a systematic review. Reprod Biol Endocrinol 2014;12:26. 


\section{Figure Legends}

Figure 1.

Normative model for the non-growing follicle (NGF) population. The best model for the establishment of the NGF population after conception, and the subsequent decline until age at menopause. The figure shows the dataset $(n=325)$, the model, the $95 \%$ prediction limits of the model, and the $95 \%$ confidence interval for the model. The horizontal axis denotes age in months up to birth at age zero, and age in years from birth to 51 years. Reprinted with permission from Wallace WH and Kelsey TW. PLoS One 2010; 5: e8772. [10].

Figure 2.

Representation of the effects of gonadotoxic treatment on the ovary. The number of primordial and early growing follicles in a healthy ovary is reduced by some chemotherapy regimens, with additional effects on the ovarian vasculature and stroma. If a sufficient population of primordial and thus early growing follicles remains, development of preovulatory follicles will continue allowing the potential for post-treatment fertility. Otherwise, complete depletion results in POI, infertility and estrogen deficiency. Ongoing post-treatment ovarian function may develop into $\mathrm{POI}$, depending on the remaining ovarian reserve.

Figure 3.

Schematic of the effect of gonadotoxic cancer treatment on ovarian function. The 3 lines represent women with high, average and low ovarian reserve (as e.g. reflected in $\mathrm{AMH}$ concentrations). Treatment results in a rapid fall in all women. Those with low ovarian reserve (red line) are more likely to develop POI during treatment, and for that to persist thereafter. Conversely, those with higher ovarian reserve will show a variable recovery, some going on to develop early POI (blue line) with others, at the highest level of ovarian reserve (green line) showing more prolonged ovarian activity, with later the normal agerelated decline.

Figure 4. 
Prognostic mosaic chart using age and AMH to predict menses $(\mathrm{M})$ or amenorrhoea $(\mathrm{A})$ at 25 years post-treatment in women with early breast cancer (reproduced with permission from Anderson RA, et al. Eur J Cancer 2013; 49: 3404-3411)[36]. 